\title{
Understanding the Effects of Control and Transparency in Searching as Learning
}

\author{
Cecilia di Sciascio \\ Eduardo Veas \\ Know-Center $\mathrm{GmbH}$ \\ Graz, Austria \\ \{cdisciascio,eveas\}@know-center.at
}

\author{
Jordan Barria-Pineda \\ Colleen Culley \\ University of Pittsburgh \\ Pittsburgh, USA \\ \{jab464,cmc98\}@pitt.edu
}

\begin{abstract}
In this paper, we analyze the benefits of adopting user interfaces that offer control and transparency for searching in contexts of learning activities. Concretely, we conducted a user study with pharmacy students performing a problem-solving task in the course of a university lecture. The task involved finding scientific papers containing relevant information to solve a clinical case. Students were split into two independent groups and assigned one search tool to perform the task. The baseline group worked with PubMed, a popular search engine in the life sciences domain, whereas the second half of the class was assigned an exploratory search system (ESS) designed for control and transparency. In the analysis, we cover the objective and subjective dimensions of the task outcomes. Firstly, the objective analysis addresses the inherent difficulty of the search task in a learning scenario and identifies certain improvements in performance for those students using the ESS, most notably when searching for primary-source content. The subjective analysis investigates the human factors side, providing evidence that the ESS effectively increases the perception of control and transparency and is able to produce a better user experience. Lastly, we report on perceived learning as a subjective dimension measured separately from user experience.
\end{abstract}

\section{CCS CONCEPTS}

- Information systems $\rightarrow$ Web searching and information discovery; Search interfaces; • Applied computing $\rightarrow$ Interactive learning environments.

\section{KEYWORDS}

Exploratory Search, Searching as Learning

\section{ACM Reference Format:}

Cecilia di Sciascio, Eduardo Veas, Jordan Barria-Pineda, and Colleen Culley. 2020. Understanding the Effects of Control and Transparency in Searching as Learning. In 25th International Conference on Intelligent User Interfaces (IUI '20), March 17-20, 2020, Cagliari, Italy. ACM, New York, NY, USA, 12 pages. https://doi.org/10.1145/3377325.3377524

Permission to make digital or hard copies of all or part of this work for personal or classroom use is granted without fee provided that copies are not made or distributed for profit or commercial advantage and that copies bear this notice and the full citation on the first page. Copyrights for components of this work owned by others than ACM must be honored. Abstracting with credit is permitted. To copy otherwise, or republish, to post on servers or to redistribute to lists, requires prior specific permission and/or a fee. Request permissions from permissions@acm.org.

IUI '20, March 17-20, 2020, Cagliari, Italy

(C) 2020 Association for Computing Machinery.

ACM ISBN 978-1-4503-7118-6/20/03 ..\$15.00

https://doi.org/10.1145/3377325.3377524

\section{INTRODUCTION}

Search tasks with learning or investigative purposes entail a combination of querying and browsing actions, occurring as part of a dynamic process often called exploratory search [42]. Exploratory search is usually motivated by a complex information problem, where the user initially lacks or has little understanding of relevant terminology or information space structure [75]. Thus, it is by definition open-ended: the user starts searching with a broad idea of the topic in mind and becomes familiar with new terminology as the task progresses, which enables them to filter out irrelevant content and spot potentially relevant resources. Such process requires careful inspection of at least a few titles and abstracts (sometimes even full-text documents), before becoming familiar with the underlying topic. Exploratory search is better supported by browsing search strategies, which promote on-the-fly selections, navigation and trial-and-error [42]. Searching as learning is a related line of research with a strong focus on measuring knowledge gain and understanding how the user learns as a result of their search behavior.

In spite of the advances in retrieval and recommender algorithms, real-world interfaces have remained largely unchanged: the user has to type a query in a text input area and results are delivered in a relevance-ranked list. In this work, we analyze the impact of exploratory search systems designed for control and transparency with respect to traditional information retrieval systems, in the context of a searching as learning scenario. We leverage an Exploratory Search System (ESS) inspired by research on human factors of recommender systems. On one hand, user-controlled recommending interfaces are preferred over static ones because users perceive that the system is able to grasp their needs better [43]. On the other hand, explanatory interfaces increase trust in the system because they convey how the system works in a transparent manner [30,68].

Taking the ESS as a benchmark tool, we conducted a user study with 115 pharmacy students, who were asked to address a realworld clinical case with the assistance of a search tool. Solving the task implied that students had to acquire domain knowledge in the course of the search session. In a nutshell, the comprehensive analysis presented in this paper contributes to understanding the effects of a system like the ESS on multiple aspects defined in the following research questions:

RQ1. Learner Performance: Can the ESS bridge the knowledge gap inherent to a searching as learning task? That is to say, we seek to assess whether the search tool contributes to objective knowledge gain. We further inquire whether learner performance stays uniform or varies for different types or literature resources. 
RQ2. User Experience: Do learners searching with the ESS perceive increased control and transparency in the system? How do these subjective system traits affect user satisfaction? In addition, what is the influence of the learner' self-assessed expertise?

RQ3. Perceived Learning: Do students perceive they gain knowledge as a result of their own search behavior or does the search system play a role? Is there a correlation with objective learning? Finally, is user experience an indicator of perceived learning?

\section{RELATED WORK}

We review the design and evaluation of control and transparency features in recommender and search systems, followed by an overview of searching as learning and gaps addressed in our study.

\subsection{Control and Transparency}

2.1.1 Recommender Systems. The recommender systems (RS) community acknowledged the need for user-centric design and evaluation approaches much sooner than the area of information retrieval, motivated by trust issues in black-box recommendation models and the lean-back nature of this information access paradigm [47, 64] RS do not only have to fetch items that match a profile of the target user, but also persuade the user to try the recommended items [67] Thus, the UI must deliver recommendations in a way that they can be compared and explained [57]. Early approaches looked into increasing interaction by enabling the user to provide preference feedback [12], though with a focus in alleviating the cold start problem rather than improving how the user perceives the interaction with the RS. Several studies sustain the multidimensional nature of the user experience $[40,68]$ and shed light on some key aspects to consider in the design of recommending interfaces: (i) granting the user more control over recommendation algorithms [22, 28, 38, 43], (ii) explaining how the system works and makes decisions (transparency) [30,68], (iii) allowing users to tell the system when it is wrong (scrutability) [34] or (iv) enabling users to predict the effect of their interaction [33]. In the case of control and transparency, these traits are often sought by way of visual and interactive mechanisms, e.g. to elicit user preference and explain the recommendation process [11], tweak and assess the influence of neighbors in collaborative filtering [46], depict relationships among multiple agents in social RS [74] or control hybrid recommendations [51].

In order to determine the impact of algorithms and user interface (UI) design decisions, some metrics and evaluation frameworks to measure the user experience with RS in terms of their attitudes and behavior were proposed $[40,55]$. Particularly, the framework from Knijnenburg et al. [39] (applied in our analysis of UX in section 4.3) describes how subjective traits that users perceive in the system affect the user experience, e.g. in terms of satisfaction with the system, with their choices or with the ease of the task or process.

2.1.2 Exploratory Search Systems. Some approaches to support exploratory search seek to dynamically adapt the workings of the search engine in function of whether the user behavior is predicted to be exploratory or lookup [5]. Another line of research applies visualizations and interactions to enrich the user interface, thereby relying on the user to actively steer the search process. These search UIs often provide some sort of relevance-to-query explanations to augment ordered lists $[29,31,56,59,66]$ or using spatial layouts that exploit reference points, such as query terms, keywords or concepts and convey relevance by arranging documents around them [1, 2, 48]. More recent approaches towards fluid information exploration seek to provide hypercues linking entities in the information space [36], employing techniques such as interactive intent modeling of user profiles [33, 60, 61], visual re-ranking in spatial relevance maps [37]. Also, specific interactions for touch devices have been applied to search interfaces [35].

The benchmark system employed in this work builds upon the uRank system [18, 19], which promotes a user-driven approach influenced by work on control and transparency in interactive RS. uRank enables users to discover and refine their search interests by way of interactions with extracted keywords and provides a visual ranking metaphor to explain document relevance with respect to evolving information needs. User studies report lower cognitive workload, as well as similar relevance assessments for subjects working with $u$ Rank and a baseline SERP condition $[18,19]$.

In addition, streams of work on social search created new opportunities to enhance control and transparency in the search interface. Social search is the common name for a group of information retrieval approaches that leverage traces left behind by past users of information systems to help future users in the search process $[23,25]$. Unlike RSs, social search is intrinsically shaped by information-seeking behavior [13]. Displaying social information helps to enrich the search process, for example by augmenting results with social cues $[24,49,65]$ or visualizing social links [20]. Also, the social-enhanced version of $u$ Rank applies control mechanisms and fine-grained explanations for multiple sources of relevance $[16,17]$. Allowing users to decide how content-based and social methods influence the final document ranking has a positive effect on transparency, user experience and system performance. The scope of this work is not to introduce novel features but rather to investigate whether an ESS offering control and transparency in a context of searching as learning has an impact on objective and subjective learning, as well as on the user experience.

\subsection{Searching as Learning}

Collecting information about a new topic is rarely solved with a single query [45]. It is instead a discovery process, which results in one building a body of knowledge, a connected collection of resources associated by meaning mostly as a cognitive construct [73]. Pirolli conceptualizes this as sense-making: finding and collecting information from large information collections, organizing and understanding it, and producing some product [54]. The way the exploration and sense-making unfolds, processing chunk after chunk of information, has been formalized in theories such as the berry picking model [8], or information foraging theory [52, 53]. These models acknowledge the fact that user needs continually shift in the process of reading and learning from the information encountered, and propose different ways to model changes in information needs. In turn, exploratory search distinguishes two kinds of behavior, lookup and browsing [42], which unfold in the course of learning or investigative tasks. As for searching as learning, Rieh et al. [58] propose the concept of comprehensive search to describe the learning that occurs during iterative, reflective and integrative search sessions. Comprehensive search encompasses exploratory search 
in the sense that both accomplish receptive and critical learning as an outcome of the search process [58]. However, comprehensive search also considers the achievement of creative learning.

Research on searching as learning has a strong focus on measuring knowledge gain and understanding how the user learns as a result of their search behavior. Eickhoff et al. [21] investigated how people's behavior changes over the course of a search session and proposed metrics to differentiate between declarative and procedural search sessions. They showed that document features obtained through a session correlate with learning intent. The learning resulting from a search session can be characterized as knowledge gain. Collins-Thompson et al. [15] investigated potential indicators of learning. They found that query interaction conditions and presentation of results are associated with achieving combined factual and conceptual knowledge gains. Gadiraju et al. [26] used knowledge tests to calibrate knowledge before and after a search session, thus quantifying knowledge gain. Yu et al. [76] followed up extracted features from logged interactions during the search and deployed standard machine learning models to predict knowledge gain, albeit with .43 accuracy. They found their used features to be weakly correlated with knowledge gain. One issue with using prior-knowledge tests is that participants learn from the tests themselves. Gadiraju et al. found in their study, that participants used terms from the knowledge test to search, effectively steering the information need [26]. This body of work shows that Searching as Learning has gained attention as a research area by itself $[58,70]$.

2.2.1 Shortcomings in Searching as Learning Studies. Based on the reviewed literature, we identified a series of gaps or shortcomings in studies of searching with the purpose of learning.

The influence of the search system in learning outcomes: Most studies have used conventional web search systems as test platforms, e.g. general-purpose search engines [21] and academic bibliographic databases [72]. The specific system traits that could support or undermine the search process seem to be overlooked. Beyond task complexity and the searcher's own expertise, the characteristics of the user interface constitute one of the major factors that influence the search tactics implemented by a searcher $[9,44]$. The UI determines the available information at a time and the actions that the user can perform on it. Indeed, further research is needed to support effective and richer user interactions in search systems used as tools.

Multi-prospect comparative analysis: out of the three aspects covered in our research questions, learner performance and subjective learning are frequently measured in searching as learning studies, albeit in a single condition rather than in A/B tests. Exploratory search studies often investigate user performance in comparative settings against a baseline [18, 27, 37], though rarely measured as a learning outcome. Perceived learning is not a concept visited in exploratory search. Lastly, UX is largely evaluated for RS [38, 39] and lately social exploratory search [16, 17], but not for the case of searching as learning.

Ecological validity: some conditions that contribute to ecological validity of user studies are often missing, e.g. the lack of a strong baseline condition. Most studies on exploratory search and RS use as baseline a knock-out version of the full system. Sampling representative subjects of the target population and defining a real-world task are crucial to foster inherent motivation in the subjects. Lastly, a sufficiently large sample size is important to convey generalizable observations. In searching as learning we find studies with samples roughly between 20 and 40 subjects, e.g. [71, 72].

In order to try to overcome most of the aforementioned gaps, we executed the study in a classroom setting with 115 pharmacy students, who conducted a search task to find evidence for a clinical treatment. Half of the students worked with an interactive ESS that offers search control and transparency features and the other half with the PubMed system. The realistic nature of the task, the representativeness of the subjects and the relatively large sample size promote near-intrinsic motivation.

\section{THE EXPLORATORY SEARCH SYSTEM (ESS)}

For this study we replicated functionalities of $u$ Rank $[18,19]$, a user-driven exploratory search tool [18], which promotes control and transparency in the UI. Control refers to the possibility for the user to influence the decisions of the system, whereas transparency is the ability of the system to explain to the user how it works. The system offers mechanisms to elicit information needs and re-rank search results on-the-fly, complemented with transparent graphic representations of relevance-to-query scores. In the remainder, we refer to this system as ESS. ESS automatically extracts keywords from an initial set of retrieved search results. In order to enable the discovery of multi-term keywords, often called key phrases, we extended the process to identify noun-phrase chunks in sentences. Figure 1 illustrates the user interface (UI) of ESS, which displays a list of search results (left panel) along with the extracted keywords organized in a word-cloud fashion (right panel). The visual arrangement of keywords is meant to provide an overview of the "aboutness" of the entire set of results at a glance.

\subsection{Controlling the Ranking}

The system relies on interactions with extracted keywords (unigrams as well as multi-term) to build a short-term model of the user's information needs. The visual query mechanism allows the user to select single terms, adjust their weights and remove them from the resulting query. Adding terms manually by typing in the text input atop the cloud is possible as well. User selected keywords (and their weights) are the inputs of a content-based (CB) model that re-ranks results in real-time. (For further details refer to [19]). A previous version of the tool [18] made it possible to visualize and select co-occurring terms in a two-step mechanism (combination of clicks and drag-and-drop). The shortcoming was that co-occurring terms appeared dispersed in the tag cloud and that it was not clear which multi-term phrases actually appeared in the text. Instead, the discovery of key phrases is simplified by listing suggestions in a tooltip when the user hovers with the mouse pointer over a particular term. Displaying single terms and showing related key phrases on-demand helps avoid clutter in the keyword cloud. Finally, the document ranking can be filtered by setting the time brush widget.

\subsection{Explaining the Ranking}

Selected keywords are arranged at the top of the UI in the query box. Interactions with keyword are considered expressions of interest and thus trigger changes in the ranking view (center panel). 


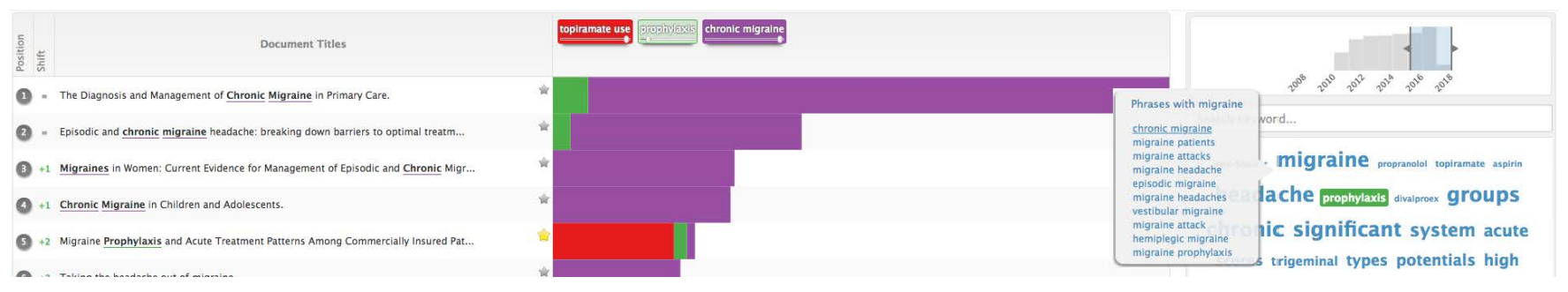

Figure 1: User Interface of the ESS

Documents are dynamically ranked in relation to the chosen terms and augmented with color-coded stacked bars to reflect keyword contribution to the overall score. Clicking on an item list opens a document viewer, showing title and abstract with keywords highlighted in the corresponding color. As a design principle, color encoding and stacked bars aim at providing transparency with respect to ranked results.

\section{USER STUDY: SEARCHING AS LEARNING}

In this study, we aim to assess the impact of working with the ESS in a searching as learning scenario. Pharmacy students had to conduct a search session in order to solve a knowledge-based task relevant to their domain. We organize the analysis according to three pillars: (i) user performance, which concerns the quality of information that students determine is relevant to solve the task; (ii) user experience, addressing the reach of control and transparency features from an affective perspective; and (iii) perceived learning, measuring the amount of knowledge that students feel have gained.

\subsection{Methodology}

In cooperation with the School of Pharmacy of University of Pittsburgh, we recruited novice pharmacy students in their first professional year of the Doctor of Pharmacy degree program. We conducted the study during a session of the Pharmacist Patient Care 1 course. As part of their training, students need to learn how to search and identify scientific literature relevant to address medication-related questions.

4.1.1 Task Goal and Expected Product. The task scenario was set in the context of a hypothetical patient seeking treatment for prevention of migraines. The patient's physician asks the pharmacy students to determine if a specific medication, metoprolol, would be appropriate therapy to prevent migraines for this patient. Students had to ponder several pieces of information, such as the patient's history of present illness and current medications, to find relevant evidence with the assigned search tool to answer the question at hand. Appendix A discloses the the task scenario and question.

Concretely, we asked students to collect a total of six bibliographic references containing key information for the case, of which three had to be primary sources and the other three tertiary sources. Primary sources refer to published original observations or experiments. Secondary sources are searchable databases used to locate published primary and tertiary resources. Lastly, tertiary sources filter and summarize primary literature on a topic, e.g. books, practice guidelines, systematic reviews. Learning to identify and apply the different kinds of sources of information is a fundamental part of the training for future pharmacists to make critical decisions.

Note that students did not have to write a response to the task question. Together with the lecturer and for the sake of a uniform assessment, we assume that students able to identify the right resources would have acquired useful knowledge to provide a recommendation for the case. Hence, performance assessment was based on the relevance of their collected references.

4.1.2 Baseline System. We employed PubMed ${ }^{1}$ for the baseline condition. PubMed is a free secondary resource that primarily accesses the MEDLINE ${ }^{2}$ database, which maintains references and publications in life science domains with a concentration on biomedicine. PubMed is the de facto search engine that pharmacy students are taught to use for investigating medication-related topics. Using PubMed, students in the baseline condition could bookmark resources into collections and download them as text files.

4.1.3 System Preparation. Using a crawler script, we pre-fetched all documents related to migraine from the Entrez e-utilities ${ }^{3}$, which are provided by NCBI (National Center for Biotechnology Information) to query PubMed's database. We processed retrieved items with the keyword extraction module and stored them in a database index. Upon user interactions with keywords, the ESS retrieves documents containing the selected terms and creates the ranked list displayed in the UI. Participants could bookmark resources during the session and download them in textual format.

4.1.4 Participants. A total of 115 pharmacy students from the School of Pharmacy PittPharmacy (University of Pittsburgh School of Pharmacy) in the first semester of their first professional year took part in the study. We consider their motivation to participate was near-intrinsic, since the task scenario matches their domain of interest. As part of their education, they are expected to develop analytical skills to solve complex problems, which often requires extensive literature research. Key demographic details for the sampled students are summarized below:

- Age: 8 younger than 20, 107 aged 20-29.

- Sex: 70 female, 45 male.

- Country of Birth: 102 from USA, 13 from other countries.

- Proficiency in English language: 100 native speakers, 14 advanced, 1 intermediate.

\footnotetext{
${ }^{1}$ https://www.ncbi.nlm.nih.gov/pubmed/

${ }^{2}$ https://www.nlm.nih.gov/bsd/pmresources.html

${ }^{3}$ https://www.ncbi.nlm.nih.gov/books/NBK25501/
} 
4.1.5 Procedure. The study was executed as part of a regular lecture session. The instructor of the lecture Pharmacist Patient Care 1 contributed with the task goal definition, assigning students to groups and evaluating their products. She also gathered materials, such as laptops, for the system and task introduction, and procured a suitable lecture space.

The study was executed according to a between-subject design split in four sessions. Each session hosted a group of students working with the same tool. The groups were organized as follows (with number of participants in brackets): (i) PubMed [29], (ii) ESS [30], (iii) PubMed [29], (iv) ESS [27]. Time slots were 50 minutes per session. Each session started with a brief introduction from the study moderator explaining the features of the corresponding search system and task goal. Students had about 30 minutes to conduct the search activity. Then they were instructed to download their bookmarked resources and prepare a text document with their top three primary and tertiary resources that they would use to answer the question. Students submitted the document in the learning management system Blackboard Learn, and spent the final minutes of the session completing a user experience survey.

\subsubsection{Measurements and Data Analysis.}

Performance Assessment. We compiled a list of unique resources based on the references produced by all students. Following a twolevel scoring scheme $(2=$ highly relevant to the question, $1=$ not as relevant to the response), the instructor rated all unique resources and indicated the appropriate type, namely primary or tertiary. Resources were not evaluated if not published and available in English. We mapped the lecturer's assessments to individual student's entries using an automatic script and computed aggregated scores per student and resource type, i.e. every student was assigned two scores. Formula 4.1.6 calculates $P$ and $T$ scores as the averaged weighted precision of a student's product, such that $r\left(d_{i}\right)$ is the rating given by the lecturer to document $d_{i}$ and $n=3$ is the number of expected resources per type. Note that we often shorten primary as $P$ and tertiary as $T$.

$$
\text { score }_{\{P, T\}}(u)=\frac{1}{n} \sum_{i=1}^{n} \frac{r\left(d_{i}\right)}{2}
$$

Performance scores serve as a proxy to an objective measure of knowledge gain and to determine how effectively the two systems support the search task (Section 4.2). Note that we did not make use of typical pre- and post-task tests to measure knowledge gain. One reason is that, according to the lecturer, students would most certainly have no pertinent previous knowledge regarding the patient case and no previous deliberate instruction on PubMed. Secondly, a similar evaluation protocol is a common practice for this lecture; therefore, we decided to maintain it.

User Experience Assessment. For the analysis of user experience (UX), we collected subjective feedback with a post-study questionnaire comprising 21 items in a 7-point Likert scale ( 1 = strongly disagree, 7 = strongly agree). We built a UX model following guidelines from the generic framework proposed by Knijnenburg et al. [39], which was designed to model and evaluate the user experience with recommender systems. Objective system aspects (OSA), represent the elements of the system that experimenters manipulate in order to expose study subjects to different conditions. Usual manipulations include using a specific algorithm and adjusting interactive or presentation features in the user interface. The obvious hypothesis is that these objective aspects have an influence on the user experience with the system $(E X P)$, i.e. $O S A \rightarrow E X P$. However, because this link is often weak or difficult to measure [14], the framework proposes that the characteristics that users perceive in the system (subjective system aspects, SSA for short) act as mediators of the resulting experience $(O S A \rightarrow S S A \rightarrow E X P)$. Additionally, personal $(P C)$ and situational characteristics $(S C)$, which are extrinsic to the system, also have an independent effect on users' perception and experience. Examples of personal characteristics are expertise, age, gender, etc. As for situational characteristics (not analyzed in this study), we can mention e.g. willingness to share private information or provide feedback in a particular setting.

Model construction based on the UX framework and validation with confirmatory factor analysis (CFA) and structural equation modeling (SEM) techniques is covered in Section 4.3.

Perceived Learning Assessment. In order to measure knowledge gain from a subjective perspective, we formulated two questions that score pre- and post-task perceived knowledge in a 7-point Likert scale ( 1 = None, 7 = A Lot), namely: (i) How much information that can be used to solve the given study case did you know beforehand, i.e. before the study session?, (ii) How much new knowledge did you gain from searching with system $[\mathrm{X}]$ ?

Section 4.4 analyzes perceived learning as the difference between pre- and post-task knowledge scores, i.e. (ii) minus (i). We consider perceived learning as a separate element from "user experience" because it is not clear how this construct fits within the generic UX framework [39]. We do, however, make use of our UX model to estimate an overall score and conduct mediation analysis between search system and perceived learning through user experience.

\subsection{Analysis of Learner Performance (RQ1)}

This section reports on the effectiveness of students at collecting relevant references for the knowledge-based task. Performance scores serve as a proxy to objective knowledge gain. So-called strict scores are computed based on (i) the relevance of the selected resource and (ii) the correct identification of its source type. We also provide a tolerant interpretation of scores, i.e. according to (i). When the type is omitted, we refer to strict scores.

The main hypothesis is that scores are overall greater for subjects working with ESS. Notwithstanding, our goal is to understand nuances in learner performance depending on resource type (primary or tertiary) and metric (strict or tolerant). We start with overall score comparisons, then break it down by resource type, reporting t-tests and Cohen's $d$ effect sizes ${ }^{4}$. Finally, we address effects all together with a mixed-effects model of (strict) performance.

4.2.1 Overview of Students' Product and Task Difficulty. Before comparing learner performance between search tools, it is important to understand the complex nature of the study task and the quality of the references fetched by students. In this part we focus on the general assessment of students' products provided by the lecturers, i.e. irrespective of the tool employed for the task.

${ }^{4}$ Cohen's $d$ effect size: $[0.2,0.5)$ is small; $[0.5,0.8)$ is medium; $\geq 0.8$ is large. 


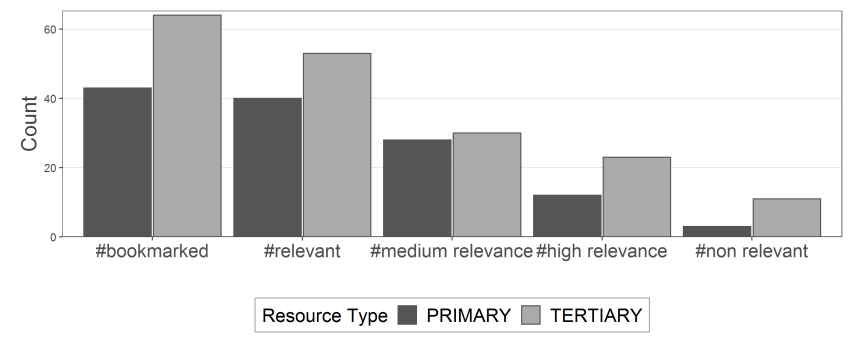

Figure 2: Relevance of unique referenced resources. The distinction between primary and tertiary is given by lecturers.

Figure 2 illustrates total unique resources collected and their relative relevance to the task. Overall, students selected 107 unique scientific articles, of which roughly $40 \%$ were primary (43 items) and $60 \%$ were tertiary (64). The overall figure represents approximately $0.4 \%$ of the total amount of retrievable articles for the query migraine in PubMed at the time of the study (October 2017). This proportion suggests that students were able to narrow down the search space into a manageable number of items with either tool.

Of the total unique references, $87 \%$ (93) were at least of medium relevance. Yet, it is evident that finding tertiary resources was more difficult, since $17 \%$ (11 items) of the references were non-relevant, in contrast to only 7\% (3 items) of the primary type (see second and last grouped bars in Figure 2). Among relevant items, about two thirds were of medium relevance ( $62 \%$ vs. $38 \%$ highly relevant). The amount of medium relevant items was much the same for the two of source types, wheres the number of highly relevant references for $T$ sources doubles the amount of $P$. In short, students found more tertiary items, but they also showed some skews towards more irrelevant and more highly relevant resources.

The quality of referenced resources was generally good. Identifying the right type of a resource proved to be more challenging. Figure 3 provides a fine-grained overview of correct and incorrect categorizations for $P$ and $T$ types. For all the short-tail items, there is a somewhat large portion of classification errors. Noticeably, the first tertiary item (the most frequently selected) was correctly classified less than $10 \%$ of the times. A cross-validation analysis reveals an overall accuracy of $71 \%$, with a one-sided test confirming that

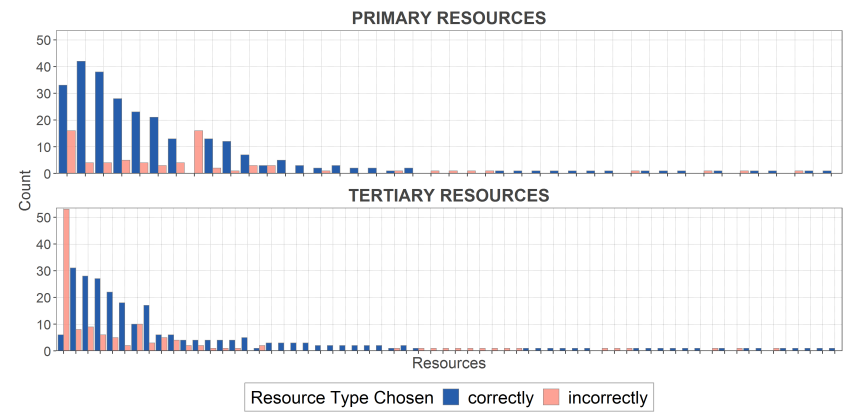

Figure 3: Frequency of correct and incorrect identification of primary and tertiary sources given by students.

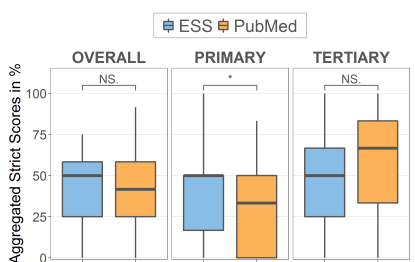

(a) Strict scores

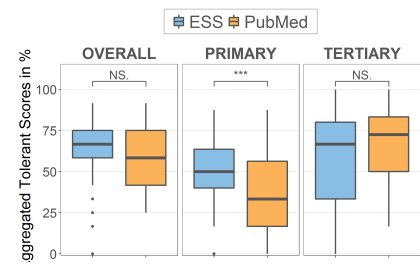

(b) Tolerant scores
Figure 4: Aggregated performance scores (with significance annotations) for overall, primary and tertiary sources.

it was significantly greater than the "no information rate" $\left(\mathrm{NIR}^{5}\right)$, $p<.001$. In other words, students' assessment was better than randomly guessing the item class. Precision was better for $T$ ( $85 \%$ vs $76 \%$ ), while recall was higher for $P$ sources (78\% vs. $65 \%)$. In the first case, students made fewer false positive decisions (i.e. lower Type I error). Identifying an item as tertiary was the right call $85 \%$ of the times. Conversely, higher recall for primary resources indicates that students identified a larger proportion of true primary sources (268 out of 343 vs 245 out of 375), which means fewer false negative errors (Type II error). Similar $\mathrm{F}_{1}$ scores, however, show that the accuracy of students' choices was balanced ( $77 \%$ for $P, 74 \%$ for $T$ ).

In summary, students efficiently produced references of at least medium relevance, but made less accurate choices regarding source types. Recall and precision varied across primary and tertiary items, though with a balanced prediction-recall trade-off. These observations intend shed light on the inherent complexity of the search task, which serves to "set the bar" prior to the comparative analysis.

4.2.2 Overall Performance. As a first estimation of overall performance, we averaged $P$ and $T$ scores across students. The left-most boxplots in Figure 4a show that it was relatively low for both search tools, roughly above $40 \%$. That is to say, approximately 2.5 out of six referenced publications were relevant to the given clinical case and the source type correctly identified. Students working with ESS achieved marginally higher scores ( $45 \%$ vs $42 \%$ ), but the difference is non significant, $t(111)=0.55, p=.58, d=0.10$.

Following a tolerant interpretation, students' scores naturally improve, with $62 \%$ for ESS against 59\% for PubMed, but the difference is again non significant, $t(111)=0.86, p=.39, d=0.16$. Interestingly, boxplots for overall performance in Figure $4 \mathrm{~b}$ show the presence of four outliers in the ESS group. After removing these four subjects, the group's scores average to $65 \%$. The difference against the PubMed group turns out significant, $t(96)=2.15, p=.03, d=0.41$, albeit with a rather small effect size.

4.2.3 Performance with Primary and Tertiary Sources. When searching for original material, students assigned to ESS outperformed the PubMed group, showing $40 \%$ average accuracy in contrast to $30 \%$ for PubMed, $t(105),=2.13 p=.03, d=0.40$. Looking at median values as a reference of data centrality ( 0.5 vs. 0.33$)$, it is clear that students missed between half and two thirds of the times. In the case of tolerant scores, students' accuracy largely improves for ESS $(M=51 \%)$ and only marginally for PubMed $(M=31 \%)$. The gap

${ }^{5} \mathrm{NIR}$ is the largest class percentage. $52 \%$ of the references correspond to true tertiary resources, thus always choosing tertiary as the source class would yield $52 \%$ accuracy. 


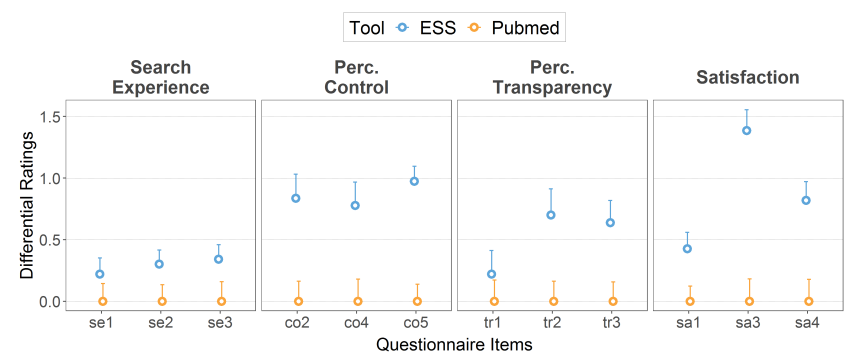

Figure 5: Responses to individual items in UX scale. PubMed scores are zeroed to show relative difference with $E S S$.

between the two systems becomes even larger (as well the effect size), $t(105)=3.45, p<.001, d=0.66$.

Conversely, students working with PubMed showed marginally better judgment for bibliography encountered in books or encyclopedia. However, the effect was non significant, $t(109)=-1.27, p=$ $.21, d=0.24$. Median values ( 0.66 both) also show the parity between average students in the two conditions. Tolerant scores reveal a larger difference in favor of PubMed, albeit non significant, $t(105)=-1.75, p=.08, d=0.33$

A possible reason for the discrepancy between $P$ and $T$ scores is that primary resources are more abundant and, being a novice searcher, it is more difficult to recognize which ones make the most substantial contributions. Therefore, it is noteworthy that ESS is able to somewhat boost students' performance in a context of higher uncertainty. ESS falling back with respect to primary resources could be attributed to the low specificity of the content provided in the abstracts of this kind of resource. Books usually cover a variety of topics, hence the abstract and the introduction may not be descriptive enough for ESS to extract terms with discriminant power. Also, sometimes document surrogates do not include any abstract or snippet for books. Therefore, fewer keywords can be extracted from them and unless the user hits exactly two or three terms in the title, these items never appear ranked near the top. Adding facet filtering by resource type could be an improvement to support novice searchers.

\subsection{Analysis of User Experience (RQ2)}

This section describes in detail the UX model built for the analysis of perceived system characteristics, overall satisfaction and the influence of personal characteristics thereon. The proposed constructs and directionality of causal effects were evaluated following the two-step approach proposed by Anderson et al. [3]: (i) fitting a measurement model to calculate factor loadings (Section 4.3.1) and (ii) fitting a structural model, which is formed by the measurement model plus causal relationships (Section 4.3.2).

4.3.1 Measurement Model of User Experience. We built the measurement model based on user responses to a post-task questionnaire comprising 21 items. We grouped items into four factors, representing constructs for: (i) Perceived Control (SSA), (ii) Perceived Transparency (SSA), (iii) Satisfaction (EXP), (iv) Search Experience (PC). A total of 9 items had to be excluded from the measurement model, 5 of them because of low communality and 5 due to high

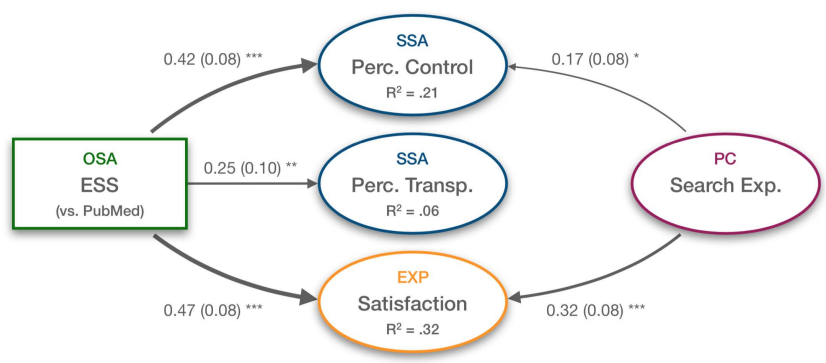

Figure 6: Structural model indicating the effect of the search system $(O S A)$ and search experience $(P C)$ on subjective system aspects $(S S A)$ and user experience $(E X P)$.

cross-loading (i.e. when they load on more than one factor). Figure 5 shows relative ratings to the questionnaire items kept in the model. The resulting model was subjected to confirmatory factor analysis with a mean- and variance-adjusted weighted least squares estimator, yielding an admissible model fit. $\chi^{2}(48)=63.23, p=.04$ $\left(\chi^{2} / d f=1.38\right)^{6} ; C F I=.99 ; T L I=.99 ; R M S E A=.05$ with $90 \% C I=[0.009, .089]^{7}$. Appendix B discloses the full scale, including item loadings to factors, factor validity and excluded items.

4.3.2 Hypotheses in Structural Regressions Model. The model in Figure 6 represents the structural regressions that correspond to the hypotheses listed below. Pathways indicate significant causal relationships, numbers and thickness denote standardized coefficients with robust standard errors.

(H1) Students will perceive more control and transparency with $\boldsymbol{E S S}(\boldsymbol{S S} \boldsymbol{A})$. Every web user is habituated to traditional interfaces displaying search results in a list. In consequence, students searching with ESS should be able to notice increased control and transparency given the variety of interactions and graphic explanations designed to that end. These causality effects are represented in the model by pathways linking the ESS OSA node with the bluestroked nodes for Perc. Control and Perc. Transp.

(H2) Students will experience higher satisfaction with ESS (EXP). The effect of the manipulated conditions (OSA) should be reflected as a more enjoyable experience (EXP). As already mentioned, it is not always possible to detect this effect directly, but it can be measured indirectly through the user's perception of the system characteristics. In this case, an indirect effect means that students are more satisfied with ESS because they perceive it as more controllable and transparent than PubMed.

(H3) Search experience will play a role in user perception and satisfaction $(P C)$. Previously acquired skills accessing bibliographic material is likely to have a role in students' to the characteristics that make ESS different from PubMed, and in turn they will be more satisfied with it.

\footnotetext{
${ }^{6} \chi^{2}$ test should be non-significant. However, in practice this is not often the case. An alternative metric is the ratio between Chi-squared statistic and degrees of freedom. $\chi^{2} / d f$ should be $<3$ (good fit) ot $<2$ (great fit).

${ }^{7}$ Acceptable cut-off values: $C F I>.96, T L I>.95$, RMSEA $<.05$ with upperbound of $90 \%$ CI $<.10$
} 
4.3.3 Results. We fit the structural model with a mean- and varianceadjusted weighted least squares estimator, which again yielded an admissible model fit, $\chi^{2}(58)=79.13, p=.03\left(\chi^{2} / d f=1.36\right)$; $C F I=.99 ; T L I=.99 ;$ RMSEA $=.05$ with $90 \% C I=[0.016, .086]$. The model in Figure 6 is the cornerstone to validate our hypotheses regarding user experience. Regression pathways report standardized coefficients $(\beta)$ and robust standard errors.

Perceived Control and Transparency. The SR model revealed that the employed tool had a positive significant effect on the two subjective system traits $(O S A \rightarrow S S A)$ stated in (H1). Students who searched with ESS indeed sensed that it was more controllable and transparent than PubMed, as it can be observed in Figure 5. In other words, perceiving more control denotes that ESS users feel the system understands their information needs better, while perceiving more transparency indicates that it is clearer to them how ESS makes relevance decisions. Students perceived an increase of about $42 \%$ and $25 \%$ (in standard deviation units) on control and transparency with ESS. These outcomes shed light on the usefulness of features such as keyword-based navigation and visual ranking representations as a means to provide two-way understanding between the system and the user.

Previous studies analyzing perceptual factors in information access systems tested causality effects of one SSA over another SSA. For example, Knijnenburg et al. [38] hypothesized that perceived inspectability in interactive recommender systems influences the amount o perceived control. The UX model in di Sciascio et al. [17] considers that perceived control in social-enhanced search systems contributes to increase the perception of transparency (Perc.Control $\rightarrow$ Perc.Transp.). We included the latter relationship in the saturated model (following the two-step approach to fit structural regressions model [3]). In this case, however, perceived control does not affect transparency. That is to say, higher perceived control and transparency are independent effects that arise as the consequence of applying the manipulated condition (OSA).

Satisfaction. The employed search tool also had a significant positive effect on user satisfaction, as denoted in the SR diagram. Figure 5 illustrates that broken-down ratings were substantially higher for ESS across all items. The SR model measures the influence of the search system on user satisfaction as direct $(O S A \rightarrow E X P)$ and indirect effects through perceived system traits $(O S A \rightarrow S S A \rightarrow E X P)$. Somewhat surprisingly, higher perception of control and transparency did not contribute to satisfaction. Conversely, the effect of using ESS turned out to be entirely direct. Direct effects of manipulated system conditions on user experience are often hard to measure [14, 39], therefore the $47 \%$ (again in S.D. units) gain in user satisfaction is noteworthy and thus supports (H2).

Search Experience. We also observe an independent effect of the users' personal characteristics on perceived control and satisfaction. Students reporting more expertise were able to recognize the possibility of controlling the system more effectively $(P C \rightarrow S S A)$ and rated their experience with it as more satisfactory $(P C \rightarrow E X P)$. The perception of transparency, however, was not affected.

Our results align to related research on UX with RS and search systems, which reported similar effects of personal characteristics: experienced users understand advanced features better and are able to perceive more control over the system [17, 38, 69]. Specifically in information-seeking area investigated the effects of search experience and expertise (which are not necessarily the same [6]) reporting better task outcomes [6, 32], lower task completion time [62] and more effective strategies [44,50]. However, this set of studies focused on some aspect of performance, whereas our focus was to assess the effect of self-assessed expertise on different dimensions of the user experience. Our approach is similar to di Sciascio et al. [17], except that we used a proper scale construct (formed by 3 items instead of a single-item indicator).

It should be noted that the group of students working with ESS reported significantly higher search expertise than the PubMed group, $t(110)=5.97, p<.001$. Given that the participant grouping was completely random, it is strange that one group was assigned the most experienced students by chance. We believe there is a confounding effect between the search tool and reported expertise. In other words, the positive experience of working with ESS probably affected students' perception of their own expertise (OSA $\leftrightarrow \mathrm{PC})$. In further consequence, although the statistical evidence supports (H3), we should be cautious about accepting this result.

\subsection{Analysis of Perceived Learning (RQ3)}

In this section we compare students' reported knowledge with respect to the pharmacy case before and after the search task. We refer to pre-task and post-task perceived knowledge as prePK and pos $P K$, respectively. The difference between the two measures is as a proxy to perceived knowledge gain, or $P K G$ for short.

4.4.1 Hypotheses for Perceived Learning. We formulated two hypotheses, one related to the lone effect of students' search activity, and the second one considering the role of the search tool:

(H1) Students will perceive their knowledge relevant for the task increases after one search session (irrespective of the system).

(H2) Perceived learning will be greater after searching with ESS.

In addition, we explored the relationship between perceived knowledge gain and user experience with the systems (UX), as well as with performance assessment (i.e. objective learning).

4.4.2 Results. A t-test shows that overall posPK $(M=5.16, S D=$ 1.14) was significantly higher than $\operatorname{prePK}(M=2.34, S D=1.07)$, $t(227)=19.38, p<.001$. Students perceived on average $40 \%$ of knowledge gain as a result of their search activity, irrespective of the assigned system $(M=2.81, S D=1.56$ in rating difference). This provides evidence to support $\mathbf{H 1}$.

Concerning the second hypothesis, perceived knowledge gain was slightly greater with ESS $(M=2.8, S D=1.42)$ than with PubMed $(M=2.74, S D=1.69)$, but not significantly, $b=0.15, t(110)=$ $0.52, p=.62$. Students did not perceive they gained more knowledge with one tool or the other, hence $\mathbf{H} 2$ is not supported.

The two tests combined show that students indeed perceive a substantial knowledge gain after performing the task. However, this effect is most likely a result of the cognitive process led by their information-seeking behavior, which seems to be independent from the external support they use for the search activity.

4.4.3 Indirect Effect of User Experience. The effect of search tool over $P K G$ reported above corresponds to the total effect. We further investigated the influence of students' experience on subjective 


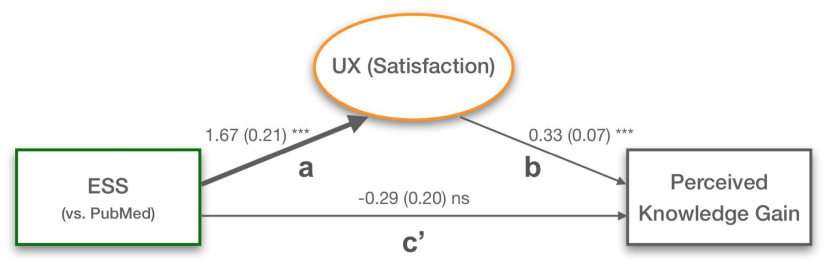

Figure 7: Path model showing indirect effect of search tool on perceived knowledge gain though user experience.

learning with mediation analysis. This technique measures whether an indirect or mediated effect exists, with $U X$ acting as an intermediate variable. A non significant total effect does not imply the lack of mediation [41] as an indirect effect could still be established.

We built the path model illustrated in Figure 7 and computed the direct effect tool $\rightarrow P K G$ and the indirect effect tool $\rightarrow \mathrm{UX} \rightarrow$ $P K G$ that add up to the total effect $\left(c=a b+c^{\prime}\right)$. This model is not connected to the one from Section 4.3, but we use it to estimate overall UX based on predicted "satisfaction", which is obtained as the linear combination between survey responses (sa1, sa3, sa4) and factor coefficients (loadings in Table 1). We fit the model with a weighted least squares estimator (WLS) and report standardized coefficients $\beta$ with standard errors and $\mathrm{p}$-values.

Path $c$ ' denotes a negative direct effect that turned out non significant, whereas paths $a$ and $b$ (the two paths composing the indirect effect) are positive and significant. Bootstrap standard errors and 95\% confidence intervals ${ }^{8}$ for the indirect effect (product of $a$ and $b$ ) corroborate that it is indeed significant, $\beta=0.38, S E=0.14, C I=$ [0.186, 0.639] (CI does not include 0 , hence $p<.05$ ). Competitive direct and indirect effects cancel each other out (opposite signs), hence the lack of total effect. In other words, even though $P K G$ is marginally better for the ESS (total effect c), path $c$ ' is negative because of the conflict with the indirect effect. Thus, we observe a positive influence of search tool over perceived learning that is entirely indirect through the students' subjective experience with the system. This type of effect corresponds to an indirect-only mediation [77] and provides evidence that partially supports $\mathbf{H} 2$.

4.4.4 A Comparison with Objective Knowledge Gain. To compare perceived learning with objective knowledge gain (using performance scores as a proxy) we do not assume causality but rather investigate whether the two variables correlate. A Pearson's productmoment test revealed that students' perception of knowledge gain does not correlate with their performance scores, $r=.03, p=.78$. Analyzing students in the ESS and PubMed groups separately, we observed that the correlation coefficient for the exploratory system is positive, $r=.10, p=.47$, but negative for PubMed, $r=-.04, p=.78$. These results were nevertheless non significant.

In order to further understand differences between perceived and objective measures, we analyzed them (in percentiles) in function of performance score quartiles. Figure 8 denotes a large gap between what students perceived and actually learned during the task Although the trend for perceived learning remains rather flat, they

${ }^{8}$ Bootstrap [63] is a resampling method that handles the non-normality of a mediatedeffect sampling distribution, i.e. the product of two coefficients [41].

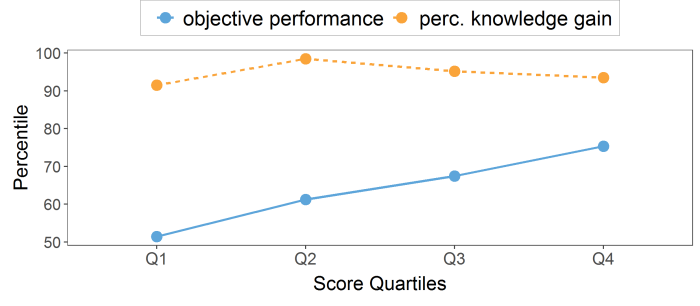

Figure 8: Perceived knowledge gain and objective performance scores as a function of score quartiles.

tended to overestimate the amount of knowledge gained across all quartiles, particularly in Q1 and Q2. This behavior occurred irrespective of the assigned tool and is not entirely surprising. Poor self-assessment skills have been observed, for example in medical or pharmacy students who over-confidently evaluated their clinical skills $[4,7,10]$. Evidently, bridging this perception gap in exploratory search systems requires further investigation and stays out of the scope of this work.

\section{OUTLOOK}

This study was designed on the basis of a natural problem-solving task that allowed for observing objective and subjective effects in a realistic searching as learning scenario. Also, the relatively large sample provides a solid ground for the drawn conclusions.

\subsection{Summary of Findings and Discussion}

RQ1. Learner Performance: Students achieved better performance with ESS in the case of primary resources and, to some extent, overall performance was slightly better according to the tolerant score interpretation. Nevertheless, general performance was in the midrange. It is difficult to underpin low effectiveness to one specific cause. Task complexity certainly played an important role. Other factors, such as insufficient engagement or objective expertise were unfortunately not measured. Nevertheless, given that the sampled students were novice searchers of scientific literature, we assume they had little expertise and that it affected their performance. In any case, the fact that a user-driven tool like ESS turned more supportive when searching for original material is to be noted.

RQ2. User Experience: The advantages of a controllable and transparent system are more tangible on the affective side. Students reported a more satisfactory experience with the ESS and acknowledged control and transparency traits as well. Considering that the baseline system is widely used in the life science domain, these results become even more relevant. It is known that search interfaces that support browsing behavior like trial-and-error selections, navigation and brushing are more apt for investigative or learning tasks. Thus, designing for control and transparency seems a promising path to support exploration as part of the learning process. In our opinion, even popular and sophisticated systems like PubMed could benefit from adding visual controls and explanations.

RQ3. Perceived Learning: Most students perceived a large difference between the knowledge they had before and after the task. Subjective knowledge gain appears as a result of search behavior, 
whereby the influence of one tool or another seems negligible. Interestingly, however, searching with the ESS contributes indirectly to greater perceived learning, so long their experience with the system is highly satisfactory. Lastly, students generally overestimate how much knowledge they acquired, irrespective of their grades.

\subsection{Limitations}

Students worked in scheduled batches with fixed time slots $(\sim 1$ hour), which set an artificial constraint. The study setup also prevented us from conducting focused observations or interviews. Regarding the participant sample, it is clear that pharmacy students have a very specific profile. Even though they were relatively novice, we cannot claim that our findings will be replicated across more diverse educational backgrounds and knowledge domains.

Finally, some limitations concern the aspects of measurement and analysis. Assessing performance via scores based on relevance and resource type judgments was the most suitable proxy to knowledge gain in the current situation. It is possible that using this ad-hoc measurements instead of typical pre/post-task tests makes our findings harder to generalize and compare with future research. With respect to the confounding effect between experimental conditions and self-assessed expertise in the analysis of user experience, we think the correct procedure to avoid this bias is to formulate the latter questions before the task begins. We understand the followed procedure limits the validity of our findings regarding the role of students' search expertise. Finally, our measurement of perceived learning was quite rudimentary. We did not build a factor-based construct ${ }^{9}$, but only used two questions to assess preand post-task perceived knowledge. Although several instruments for self-assessment of learning are available, they mostly target the classroom or virtual learning environment, e.g. MOOCs. More work is needed to develop an instrument that models the perception of learning during search activities.

\subsection{Future Work}

Students had better performance and higher satisfaction with use of the ESS compared with PubMed, the traditionally used searchable database. Continued development of search tools that will allow users to fine-tune searches by increasing the relevance of the results is welcome. The more efficient and effective the search strategy is in finding relevant literature, the better the care of the patient. In the future we intend to use metrics and features to categorize queries and quantify learning intent. These metrics can serve as basis to predict the type of information that should be relevant and to adapt the type of presentation accordingly.

\section{APPENDIX}

\section{A TASK SCENARIO}

\section{Setting: Am Care}

Scenario Introduction: Sandy Parish presents today for a well-visit to the clinic. She is being seen by the pharmacist for a review of her medications. History of Present Illness (HPI): Sandy Parish is 25-year-old white female. She is 5'3" and weighs $120 \mathrm{lb}$. She was diagnosed with migraines approximately 10 years ago. The frequency of the migraines has increased over the past year. She has experienced between 4-5 migraines per month

\footnotetext{
${ }^{9} \mathrm{~A}$ factor should be formed by at least 3 loading items.
}

over the last year. She does not typically have an aura prior to migraine. She has not been able to identify any consistent triggers for the migraines. The pain is typically moderate and pulsating in nature. She tends to find relief by resting in dark room and taking naproxen. Her concern now is that the frequency of the migraines is leading her to miss work. She just started a job as a sales consultant for a building company about 6 months ago. She was excited to be able to get a full-time job that allows her to use her degree and allowed her to have health insurance with good prescription coverage. After your interview with the patient, you learn that she does not have hypertension (her blood pressure this visit was good) or any history of heart conditions, she does not use alcohol, smoke, nor use illicit drugs. She is not currently sexually active. She is trying to maintain healthy habits such as getting enough sleep, eating balanced meals, and exercising. Current Medications:

\begin{tabular}{|c|c|c|c|}
\hline Medication & When Started & pharmacy Condition & When diagnosed \\
\hline $\begin{array}{c}\text { Centrum multivitamin one } \\
\text { tablet by mouth daily }\end{array}$ & 10 years ago & General Health & \\
\hline $\begin{array}{c}\left.\text { Naproxen sodium (Aleve }{ }^{\infty}\right) 220 \mathrm{mg} \\
2-3 \text { caplets by mouth as needed at } \\
\text { the onset of migraine symptoms }\end{array}$ & 10 years ago & Migraines & 10 years ago \\
\hline
\end{tabular}

Question posed to students: Dr. Patten would like to know if metoprolol might be helpful for prevention of Sandy's migraines?

\section{B USER EXPERIENCE SCALE}

Table 1 reports the full UX scale, comprising 21 items grouped in four factors. Grayed items were excluded due to low communality (high variance) or high cross-loadings. Factor fit is indicated in the left-most column, below factor names. All factors showed good convergent validity $(A V E)$, internal consistency reliability (Cronbach's $\alpha$ ) and discriminant validity (all interfactor correlations are $<.85$ ). The two right-most columns indicate item loadings to factors and their uniqueness, measured as residual variance.

Table 1: Factor and Items in User Experience Scale

\begin{tabular}{|c|c|c|c|c|}
\hline Factor & \# & Question & Loading & Var. \\
\hline \multirow{2}{*}{$\begin{array}{l}\text { Search } \\
\text { Experience } \\
\text { (PC) }\end{array}$} & se1 & How experienced are you in bibliographic search? & 0.75 & 0.45 \\
\hline & se2 & $\begin{array}{l}\text { How familiar are you with general purpose search engines } \\
\text { (e.g. Google, Bing)? }\end{array}$ & 0.65 & 0.58 \\
\hline \multirow{2}{*}{$\begin{array}{l}A V E=.57 \\
\alpha=.72\end{array}$} & se3 & $\begin{array}{l}\text { How familiar are you with biopharmacy literature search engines } \\
\text { (e.g. PubMed, RefMed)? }\end{array}$ & 0.86 & 0.26 \\
\hline & se4 & $\begin{array}{l}\text { Are you familiar with management tools for research papers } \\
\text { (e.g. Google Scholar, Mendeley, Zotero)? }\end{array}$ & & \\
\hline \multirow{3}{*}{$\begin{array}{l}\text { Perceived } \\
\text { Control } \\
\text { (SSA) }\end{array}$} & col & I felt the system had limited functionalities $(-)$ & & \\
\hline & co2 & The system allowed me to refine my search terms easily & 0.8 & 0.35 \\
\hline & $\cos 3$ & If I make an unhelpful search action in the system it is easy to revert it & & \\
\hline \multirow{4}{*}{$\begin{array}{l}A V E=.70 \\
\alpha=.84\end{array}$} & co4 & I felt that I was able to tell the system exactly what I wanted it to do & 0.90 & 0.20 \\
\hline & $\cos$ & I found the system intuitive & 0.81 & 0.35 \\
\hline & $\cos$ & $\begin{array}{l}\text { I often felt confident that the chosen keywords would help me } \\
\text { find good results }\end{array}$ & & \\
\hline & co7 & Search actions supported by the system were hard to control & & \\
\hline \multirow{4}{*}{$\begin{array}{l}\text { Perceived } \\
\text { Transp. } \\
\text { (SSA) } \\
A V E=.73 \\
\alpha=.86\end{array}$} & $\operatorname{tr} 1$ & $\begin{array}{l}\text { It was easy to understand why some documents were ranked } \\
\text { higher than others }\end{array}$ & 0.78 & 0.40 \\
\hline & $\operatorname{tr} 2$ & $\begin{array}{l}\text { The system gave me a sense of transparency } \\
\text { (i.e. I understood how documents were ranked) }\end{array}$ & 0.90 & 0.21 \\
\hline & tr3 & The system was informative about the relevance of each document & 0.91 & 0.18 \\
\hline & $\operatorname{tr} 4$ & I feel the user interface was self-explanatory & & \\
\hline \multirow{6}{*}{$\begin{array}{l}\begin{array}{l}\text { Satisfaction } \\
\text { (EXP) }\end{array} \\
\begin{array}{l}A V E=.59 \\
\alpha=.80\end{array}\end{array}$} & sa1 & The system was helpful to understand the given topic & 0.77 & 0.41 \\
\hline & $\mathrm{sa} 2$ & $\begin{array}{l}\text { The system was helpful to differentiate between primary and } \\
\text { tertiary research resources }\end{array}$ & & \\
\hline & sa3 & I see a potential benefit in using the system for research tasks & 0.67 & 0.56 \\
\hline & sa4 & I felt confident while using the system & 0.77 & 0.41 \\
\hline & sa5 & I would recommend the system to a colleague/classmate & & \\
\hline & sa6 & Working with the system was exhausting (-) & & \\
\hline
\end{tabular}

Note: $A V E$ (average variance extracted) $>.5$ indicates convergent validity. Cronbach's $\alpha>.8$ is good, $>.9$ is excellent internal consistency reliability.

\section{REFERENCES}

[1] Jae-wook Ahn and Peter Brusilovsky. 2013. Adaptive visualization for exploratory information retrieval. Information Processing and Management 49, 5 (2013), 11391164. https://doi.org/10.1016/j.ipm.2013.01.007 
[2] Jae-wook Ahn, Peter Brusilovsky, and Shuguang Han. 2015. Personalized Search Reconsidering the Value of Open User Models. In Proceedings of the 20th International Conference on Intelligent User Interfaces (IUI '15). ACM, New York, NY, USA, 202-212. https://doi.org/10.1145/2678025.2701410

[3] James C. Anderson and David W. Gerbing. 1988. Structural Equation Modeling in Practice: A Review and Recommended Two-Step Approach. Psychological Bulletin 103, 3 (5 1988), 411-423.

[4] MA Antonelli. 1997. Accuracy of second-year medical students' self-assessment of clinical skills. Academic Medicine 72, 10 (1997), S63-S65.

[5] Kumaripaba Athukorala, Dorota Głowacka, Giulio Jacucci, Antti Oulasvirta and Jilles Vreeken. 2016. Is Exploratory Search Different? A Comparison of Information Search Behavior for Exploratory and Lookup Tasks. 7. Assoc. Inf. Sci. Technol. 67, 11 (Nov. 2016), 2635âĂŞ2651. https://doi.org/10.1002/asi.23617

[6] Anne Aula. 2005. Studying user strategies and characteristics for developing web search interfaces. Ph.D. Dissertation. University of Tampere. https://tampub.uta. fi/handle/10024/67544

[7] Zubin Austin and Paul AM Gregory. 2007. Evaluating the accuracy of pharmacy students' self-assessment skills. American journal of pharmaceutical education 71, 5 (2007), 89.

[8] Marcia J. Bates. 1989. The design of browsing and berrypicking techniques for the online search interface. Online Review 13, 5 (may 1989), 407-424. https //doi.org/10.1108/eb024320

[9] Marcia J. Bates. 1990. Where should the person stop and the information search interface start? Information Processing \& Management 26, 5 (jan 1990), 575-591. https://doi.org/10.1016/0306-4573(90)90103-9

[10] Danielle Blanch-Hartigan. 2011. Medical students' self-assessment of performance: Results from three meta-analyses. Patient education and counseling 84 (07 2011), 3-9. https://doi.org/10.1016/j.pec.2010.06.037

[11] Svetlin Bostandjiev, John O Donovan, and Tobias Höllerer. 2012. TasteWeights: A Visual Interactive Hybrid Recommender System. Proceedings of the 6th ACM conference on Recommender systems - RecSys '12 (2012), 35-42. https://doi.org/ $10.1145 / 2365952.2365964$

[12] Peter Brusilovsky. 1996. Methods and techniques of adaptive hypermedia. User Modeling and User-Adapted Interaction 6, 2 (1996), 87-129. https://doi.org/10. 1007/BF00143964

[13] Peter Brusilovsky, Barry Smyth, and Bracha Shapira. 2018. Social Search. In Social Information Access, Peter Brusilovsky and Daqing He (Eds.). Springer International Publishing, 213-276. https://doi.org/10.1007/978-3-319-90092-6_7

[14] David N. Chin. 2001. Empirical Evaluation of User Models and User-Adapted Systems. User Modeling and User-Adapted Interaction 11, 1 (01 Mar 2001), 181-194. https://doi.org/10.1023/A:1011127315884

[15] Kevyn Collins-Thompson, Soo Young Rieh, Carl C. Haynes, and Rohail Syed. 2016. Assessing Learning Outcomes in Web Search: A Comparison of Tasks and Query Strategies. In Proceedings of the 2016 ACM on Conference on Human Information Interaction and Retrieval (CHIIR '16). ACM, New York, NY, USA, 163-172. https://doi.org/10.1145/2854946.2854972

[16] Cecilia di Sciascio, Peter Brusilovsky, Christoph Trattner, and Eduardo Veas. 2019. A Roadmap to User-Controllable Social Exploratory Search. ACM Trans. Interact. Intell. Syst. 10, 1, Article Article 8 (Aug. 2019), 38 pages. https://doi.org/10.1145/ 3241382

[17] Cecilia di Sciascio, Peter Brusilovsky, and Eduardo Veas. 2018. A Study on UserControllable Social Exploratory Search. In Proceedings of the 23rd International Conference on Intelligent User Interfaces (IUI '18). ACM, Tokyo, Japan, 353-364 https://doi.org/10.1145/3172944.3172986

[18] Cecilia di Sciascio, Vedran Sabol, and Eduardo Veas. 2016. Rank As You Go : User-Driven Exploration of Search Results. In Proceedings of the 21st International Conference on Intelligent User Interfaces (IUI '16). ACM, Sonoma, CA, USA, 118129. https://doi.org/10.1145/2856767.2856797

[19] Cecilia di Sciascio, Vedran Sabol, and Eduardo Veas. 2017. Supporting Exploratory Search with a Visual User-Driven Approach. ACM Transactions on Interactive Intelligent Systems 7, 4 (Dec 2017), 1-35. https://doi.org/10.1145/3009976

[20] Justin J. Donaldson, Michael Conover, Benjamin Markines, Heather Roinestad and Filippo Menczer. 2008. Visualizing Social Links in Exploratory Search. In Proceedings of the Nineteenth ACM Conference on Hypertext and Hypermedia (HT '08). Association for Computing Machinery, New York, NY, USA, 213 - 218 https://doi.org/10.1145/1379092.1379132

[21] Carsten Eickhoff, Jaime Teevan, Ryen White, and Susan Dumais. 2014. Lessons from the Journey: A Query Log Analysis of Within-session Learning. In Proceedings of the 7th ACM International Conference on Web Search and Data Mining (WSDM '14). ACM, New York, NY, USA, 223-232. https://doi.org/10.1145/2556195. 2556217

[22] Michael D. Ekstrand, Daniel Kluver, F. Maxwell Harper, and Joseph A. Konstan 2015. Letting Users Choose Recommender Algorithms : An Experimental Study. In Proceedings of the 9th ACM Conference on Recommender Systems - RecSys '15. 11-18. https://doi.org/10.1145/2792838.2800195

[23] Brynn M. Evans and Ed H. Chi. 2010. An elaborated model of social search Information Processing \& Management 46, 6 (2010), 656-678.
[24] Rosta Farzan, Maurice Coyle, Jill Freyne, Peter Brusilovsky, and Barry Smyth. 2007. ASSIST: adaptive social support for information space traversal. Proceedings of the Eighteenth ACM Conference on Hypertext and Hypermedia (2007), 199-208. https://doi.org/10.1145/1286240.1286299

[25] Jill Freyne and Barry Smyth. 2004. An Experiment in Social Search. In Adaptive Hypermedia and Adaptive Web-Based Systems, Paul M. E. De Bra and Wolfgang Nejdl (Eds.). Springer Berlin Heidelberg, Berlin, Heidelberg, 95-103.

[26] Ujwal Gadiraju, Ran Yu, Stefan Dietze, and Peter Holtz. 2018. Analyzing Knowledge Gain of Users in Informational Search Sessions on the Web. In Proceedings of the 2018 Conference on Human Information Interaction \& Retrieval (CHIIR '18). ACM, New York, NY, USA, 2-11. https://doi.org/10.1145/3176349.3176381

[27] Dorota Glowacka, Tuukka Ruotsalo, Ksenia Konuyshkova, kumaripaba Athukorala, Samuel Kaski, and Giulio Jacucci. 2013. Directing Exploratory Search: Reinforcement Learning from User Interactions with Keywords. In Proceedings of the 2013 International Conference on Intelligent User Interfaces (IUI '13). Association for Computing Machinery, New York, NY, USA, 117-128. https: //doi.org/10.1145/2449396.2449413

[28] F. Maxwell Harper, Funing Xu, Harmanpreet Kaur, Kyle Condiff, Shuo Chang, and Loren Terveen. 2015. Putting Users in Control of their Recommendations. In 2015 ACM Conference on Recommender Systems, RecSys 2015. 3-10. https: //doi.org/10.1145/2792838.2800179

[29] Marti A. Hearst. 1995. TileBars: Visualization of Term Distribution Information in Full Text Information Access. In Proceedings of the SIGCHI Conference on Human Factors in Computing Systems (CHI '95). ACM Press/Addison-Wesley Publishing Co., New York, NY, USA, 59-66. https://doi.org/10.1145/223904.223912

[30] Jonathan L. Herlocker, Joseph A. Konstan, and John Riedl. 2000. Explaining Collaborative Filtering Recommendations. In Proceedings of the 2000 ACM Conference on Computer Supported Cooperative Work (CSCW'00). Association for Computing Machinery, New York, NY, USA, 241-250. https://doi.org/10.1145/358916.358995

[31] Orland Hoeber and Xue Dong Yang. 2006. The Visual Exploration ofWeb Search Results Using HotMap. In Proceedings of the Conference on Information Visualization (IV '06). IEEE Computer Society, Washington, DC, USA, 157-165. https://doi.org/10.1109/IV.2006.108

[32] Christine Jenkins, Cynthia L Corritore, and Susan Wiedenbeck. 2003. Patterns of information seeking on the Web: A qualitative study of domain expertise and Web expertise. IT \& society 1,3 (2003), 64-89.

[33] Antti Kangasrääsiö, Dorota Glowacka, and Samuel Kaski. 2015. Improving Controllability and Predictability of Interactive Recommendation Interfaces for Exploratory Search. In Proceedings of the 20th International Conference on Intelligent User Interfaces (IUI '15). Association for Computing Machinery, New York, NY, USA, 247-251. https://doi.org/10.1145/2678025.2701371

[34] Judy Kay. 2006. Scrutable Adaptation: Because We Can and Must. In Proceedings of the 4th International Conference on Adaptive Hypermedia and Adaptive WebBased Systems (AH'06). Springer-Verlag, Berlin, Heidelberg, 11-19. https://doi. org/10.1007/11768012_2

[35] Khalil Klouche, Tuukka Ruotsalo, Diogo Cabral, Salvatore Andolina, Andrea Bellucci, and Giulio Jacucci. 2015. Designing for Exploratory Search on Touch Devices. In Proceedings of the 33rd Annual ACM Conference on Human Factors in Computing Systems (CHI '15). Association for Computing Machinery, New York, NY, USA, 4189-4198. https://doi.org/10.1145/2702123.2702489

[36] Khalil Klouche, Tuukka Ruotsalo, and Giulio Jacucci. 2018. From Hyperlinks to Hypercues: Entity-Based Affordances for Fluid Information Exploration. In Proceedings of the 2018 Designing Interactive Systems Conference (DIS '18). Association for Computing Machinery, New York, NY, USA, 401-411. https: //doi.org/10.1145/3196709.3196775

[37] Khalil Klouche, Tuukka Ruotsalo, Luana Micallef, Salvatore Andolina, and Giulio Jacucci. 2017. Visual Re-Ranking for Multi-Aspect Information Retrieval. Proceedings of the 2017 Conference on Conference Human Information Interaction and Retrieval - CHIIR '17 (2017), 57-66. https://doi.org/10.1145/3020165.3020174

[38] Bart P. Knijnenburg, Svetlin Bostandjiev, John O'Donovan, and Alfred Kobsa. 2012. Inspectability and control in social recommenders. In Proceedings of the 6th ACM conference on Recommender systems - RecSys '12. 43. https://doi.org/10. 1145/2365952.2365966

[39] Bart P. Knijnenburg, Martijn C. Willemsen, Zeno Gantner, Hakan Soncu, and Chris Newell. 2012. Explaining the user experience of recommender systems. User Modelling and User-Adapted Interaction 22, 4-5 (2012), 441-504. https: //doi.org/10.1007/s11257-011-9118-4

[40] Joseph A. Konstan and John Riedl. 2012. Recommender systems: From algorithms to user experience. User Modelling and User-Adapted Interaction 22, 1-2 (2012), 101-123. https://doi.org/10.1007/s11257-011-9112-x

[41] David P. Mackinnon and Amanda J. Fairchild. 2009. Current Directions in Mediation Analysis. Current directions in psychological science 18, 1 (feb 2009), 16. https://doi.org/10.1111/j.1467-8721.2009.01598.x

[42] Gary Marchionini. 2006. Exploratory Search: From Finding to Understanding. Commun. ACM 49, 4 (April 2006), 41-46. https://doi.org/10.1145/1121949.1121979

43] Sean M. McNee, Shyong K. Lam, Joseph A. Konstan, and John Riedl. 2003. Interfaces for Eliciting New User Preferences in Recommender Systems. In User 
Modeling 2003, Peter Brusilovsky, Albert Corbett, and Fiorella de Rosis (Eds.) Springer Berlin Heidelberg, Berlin, Heidelberg, 178-187.

[44] Raquel Navarro-Prieto, Mike Scaife, and Yvonne Rogers. 1999. Cognitive strategies in web searching. In 5th Conference on Human Factors \& the Web. 43-56.

[45] Mark Nolan. 2008. IA column: Exploring exploratory search. Bulletin of the American Society for Information Science and Technology 34, 4 (2008), 38-41. https://doi.org/10.1002/bult.2008.1720340410

[46] John O’Donovan, Brynjar Gretarsson, Svetlin Bostandjiev, Tobias Hollerer, and Barry Smyth. 2009. A Visual Interface for Social Information Filtering. In 2009 International Conference on Computational Science and Engineering. IEEE, 74-81. https://doi.org/10.1109/CSE.2009.26

[47] John O'Donovan and Barry Smyth. 2005. Trust in recommender systems. Proceedings of the 10th international conference on Intelligent user interfaces - IUI '05 (2005), 167. https://doi.org/10.1145/1040830.1040870

[48] Kai A. Olsen, Robert R. Korfhage, Kenneth M. Sochats, Michael B. Spring, and James G. Williams. 1993. Visualization of a document collection: The vibe system. Information Processing Management 29, 1 (1993), 69 - 81. https://doi.org/10. 1016/0306-4573(93)90024-8

[49] Valeria Orso, Tuukka Ruotsalo, Jukka Leino, Luciano Gamberini, and Giulio Jacucci. 2017. Overlaying social information: The effects on users' search and information-selection behavior. Information Processing and Management 53, 6 (2017), 1269-1286. https://doi.org/10.1016/j.ipm.2017.06.001

[50] Ruth A. Palmquist and Kyung-Sun Kim. 2000. Cognitive style and on-line database search experience as predictors of Web search performance. Fournal of the American Society for Information Science 51, 6 (2000), 558-566. https://doi.org/ 10.1002/(SICI)1097-4571(2000)51:6<558::AID-ASI7>3.0.CO;2-9

[51] Denis Parra, Peter Brusilovsky, and Christoph Trattner. 2014. See What You Want to See: Visual User-driven Approach for Hybrid Recommendation. In Proceedings of the 19th International Conference on Intelligent User Interfaces (IUI '14). ACM, New York, NY, USA, 235-240. https://doi.org/10.1145/2557500.2557542

[52] Peter Pirolli. 2007. Cognitive Models of HumanâĂŞInformation Interaction. In Handbook of Applied Cognition: 2nd Edition (2 ed.), Francis Durso (Ed.). Wiley \& Sons, New York, NY, USA.

[53] Peter Pirolli. 2007. Information Foraging Theory: Adaptive Interaction with Information (1 ed.). Oxford University Press, Inc., New York, NY, USA

[54] Peter Pirolli and Stuart Card. 2005. The sensemaking process and leverage points for analyst technology as identified through cognitive task analysis. (2005), 2-4.

[55] Pearl Pu and Li Chen. 2011. A User - Centric Evaluation Framework for Recommender Systems. Proceedings of the 5th ACM conference on Recommender systems - RecSys '11 (2011), 157-164. https://doi.org/10.1145/2043932.2043962

[56] Harald Reiterer, Gabriela Tullius, and Thomas M. Mann. 2005. Insyder: a contentbased visual-information-seeking system for the web. International fournal on Digital Libraries 5, 1 (2005), 25-41. https://doi.org/10.1007/s00799-004-0111-y

[57] Francesco Ricci, Lior Rokach, Bracha Shapira, and Paul B. Kantor. 2010. Recommender Systems Handbook (1st ed.). Springer-Verlag New York, Inc., New York, NY, USA.

[58] Soo Young Rieh, Kevyn Collins-Thompson, Preben Hansen, and Hye-Jung Lee. 2016. Towards searching as a learning process: A review of current perspectives and future directions. Journal of Information Science 42, 1 (2016), 19-34. https: //doi.org/10.1177/0165551515615841

[59] Jonathan Roberts, Nadia Boukhelifa, and Peter Rodgers. 2002. Multiform glyph based web search result visualization. In Proceedings of the Sixth International Conference on Information Visualisation. IEEE Comput. Soc, 549-554. https: //doi.org/10.1109/IV.2002.1028828

[60] Tuukka Ruotsalo, Giulio Jacucci, Petri Myllymäki, and Samuel Kaski. 2015. Interactive Intent Modeling: Information Discovery Beyond Search. Commun. ACM 58, 1 (2015), 86-92. https://doi.org/10.1145/2656334

[61] Tuukka Ruotsalo, Jaakko Peltonen, M. Eugster, Dorota Głowacka, Ksenia Konyushkova, Kumaripaba Athukorala, Ilkka Kosunen, Aki Reijonen, Petri Myllymäki, Giulio Jacucci, and Samuel Kaski. 2013. Directing exploratory search with interactive intent modeling. In Proceedings of the 22nd ACM international Conference on Information and Knowledge Management. 1759--1764. https://doi.org/2505644

[62] Hitomi Saito and Kazuhisa Miwa. 2001. A cognitive study of information seeking processes in the WWW: the effects of searcher's knowledge and experience. In Proceedings of the Second International Conference on Web Information Systems Engineering. IEEE Comput. Soc, 321-327. https://doi.org/10.1109/WISE.2001. 996493

[63] Patrick E. Shrout and Niall Bolger. 2002. Mediation in experimental and nonexperimental studies: New procedures and recommendations. Psychological Methods 7 , 4 (2002), 422-445.

[64] Rashmi Sinha and Kirsten Swearingen. 2002. The Role of Transparency in Recommender Systems. In CHI '02 Extended Abstracts on Human Factors in Computing Systems (CHIEA '02). Association for Computing Machinery, New York, NY, USA, 830-831. https://doi.org/10.1145/506443.506619

[65] Barry Smyth, Evelyn Balfe, Jill Freyne, Peter Briggs, Maurice Coyle, and Oisin Boydell. 2004. Exploiting Query Repetition and Regularity in an Adaptive
Community-Based Web Search Engine. User Modeling and User-Adapted Interaction 14, 5 (2004), 383-423. https://doi.org/10.1007/s11257-004-5270-4

[66] Anselm Spoerri. 2004. MetaCrystal: Visual Interface for Meta Searching. In CHI '04 Extended Abstracts on Human Factors in Computing Systems (CHI EA '04). ACM, New York, NY, USA, 1558. https://doi.org/10.1145/985921.986134

[67] Kirsten Swearingen and Rashmi Sinha. 2001. Beyond Algorithms: An HCI Perspective on Recommender Systems. In Proceedings of the ACM SIGIR 2001 Workshop on Recommender Systems. ACM Press, 1-11.

[68] Nava Tintarev and Judith Masthoff. 2012. Evaluating the Effectiveness of Explanations for Recommender Systems. User Modeling and User-Adapted Interaction 22, 4-5 (Oct. 2012), 399-439. https://doi.org/10.1007/s11257-011-9117-5

[69] Chun-Hua Tsai and Peter Brusilovsky. 2018. Beyond the Ranked List: User-Driven Exploration and Diversification of Social Recommendation. In 23rd International Conference on Intelligent User Interfaces (IUI '18). ACM, New York, NY, USA, 239-250. https://doi.org/10.1145/3172944.3172959

[70] Pertti Vakkari. 2016. Searching as learning: A systematization based on literature. Journal of Information Science 42, 1 (2016), 7-18. https://doi.org/10.1177/ 0165551515615833

[71] Pertti Vakkari and Saila Huuskonen. 2012. Search effort degrades search output but improves task outcome. Fournal of the American Society for Information Science and Technology 63, 4 (2012), 657-670. https://doi.org/10.1002/asi.21683

[72] Pertti Vakkari, Mikko Pennanen, and Sami Serola. 2003. Changes of search terms and tactics while writing a research proposal: A longitudinal case study. Information Processing \& Management 39, 3 (2003), 445 - 463. https://doi.org/10. 1016/S0306-4573(02)00031-6

[73] Eduardo Veas and Cecilia di Sciascio. 2015. Interactive Preference Elicitation for Scientific and Cultural Recommendations. In Proceedings of the 2015 International Conference on Constraints and Preferences for Configuration and Recommendation and Intelligent Techniques for Web Personalization - Volume 1440 (CPCR+ITWP '15). CEUR-WS.org, Buenos Aires, Argentina, 12-15.

[74] Katrien Verbert, Denis Parra, Peter Brusilovsky, and Erik Duval. 2013. Visualizing recommendations to support exploration, transparency and controllability. Proceedings of the 2013 International Conference on Intelligent User Interfaces - IUI '13 (2013), 351. https://doi.org/10.1145/2449396.2449442

[75] Ryen W. White and Resa A. Roth. 2009. Exploratory Search: Beyond the QueryResponse Paradigm. Synthesis Lectures on Information Concepts, Retrieval, and Services 1 , 1 (jan 2009), 1-98. https://doi.org/10.2200/S00174ED1V01Y200901ICR003

[76] Ran Yu, Ujwal Gadiraju, Peter Holtz, Markus Rokicki, Philipp Kemkes, and Stefan Dietze. 2018. Predicting User Knowledge Gain in Informational Search Sessions. In The 41st International ACM SIGIR Conference on Research \&\#38; Development in Information Retrieval (SIGIR '18). ACM, New York, NY, USA, 75-84. https: //doi.org/10.1145/3209978.3210064

[77] Xinshu Zhao, John\&\#xa0;G. Lynch, Qimei Chen, John Deighton served as editor served as associate editor for this Article., and Gavan Fitzsimons. 2010. Reconsidering Baron and Kenny: Myths and Truths about Mediation Analysis. Journal of Consumer Research 37, 2 (2010), 197-206. https://doi.org/10.1086/651257 